\title{
WEAK SOLUTIONS FOR A BIOCONVECTION MODEL RELATED TO BACILLUS SUBTILIS*
}

\author{
DMITRY VOROTNIKOV ${ }^{\dagger}$
}

\begin{abstract}
We consider the initial-boundary value problem for the coupled Navier-Stokes-KellerSegel-Fisher-Kolmogorov-Petrovskii-Piskunov system in two- and three-dimensional domains. The problem describes oxytaxis and growth of Bacillus subtilis in moving water. We prove existence of global weak solutions to the problem. We distinguish between two cases determined by the cell diffusion term and the space dimension, which are referred to as the supercritical and subcritical ones. In the first case, the choice of the kinetic function enjoys a wide range of possibilities: in particular, it can be zero. Our results are new even in the absence of the kinetic term. In the second case, the restrictions on the kinetic function are less relaxed: for instance, it cannot be zero but can be Fisher-like. In the case of linear cell diffusion, the solution is regular and unique provided the domain is the whole plane. In addition, we study the long-time behavior of the problem, find dissipative estimates, and construct attractors.
\end{abstract}

Key words. Bioconvection, oxytaxis, trajectory attractor, global solvability, Navier-Stokes, Keller-Segel, reaction-diffusion.

AMS subject classifications. 35Q35, 35B41, 35Q92, 76Z99, 92C17.

\section{Introduction}

Let us fix a number $T>0$ and a domain $\Omega \subset \mathbb{R}^{d}$, with $d=2,3$, which can be a bounded open set locally located on one side of its $C^{2}$-smooth boundary $\partial \Omega$ or the whole space $\mathbb{R}^{d}$ itself. In the cylinder $Q_{T}=(0, T) \times \Omega$, we consider the following set of equations:

$$
\begin{aligned}
\partial_{t} n+u \cdot \nabla n-\Delta\left(n^{m}\right) & =-\nabla \cdot(\chi(c) n \nabla c)+f(n), \\
\partial_{t} c+u \cdot \nabla c-\Delta c & =-k(c) n, \\
\partial_{t} u+u \cdot \nabla u-\Delta u+\nabla p & =-n \nabla \phi \\
\nabla \cdot u & =0 .
\end{aligned}
$$

Here $c(t, x): Q_{T} \rightarrow \mathbb{R}, n(t, x): Q_{T} \rightarrow \mathbb{R}, u(t, x): Q_{T} \rightarrow \mathbb{R}^{d}$, and $p(t, x): Q_{T} \rightarrow \mathbb{R}$ are the oxygen concentration, cell concentration, fluid velocity, and hydrostatic pressure, respectively. The scalar functions $k, \chi$, and $f$ determine the oxygen consumption rate, chemotactic sensitivity, and bacterial growth, respectively, $\phi: Q_{T} \rightarrow \mathbb{R}$ is the potential produced by the action of physical forces on the cells, and $m \geq 1$ is the nonlinear diffusion exponent. The cases $m=1$ and $f \equiv 0$ are not excluded.

The system is complemented with the no-flux boundary conditions for $n^{m}$ and $c$, and the no-slip condition for $u$,

$$
\frac{\partial n^{m}(t, x)}{\partial \nu}=0, \frac{\partial c(t, x)}{\partial \nu}=0, u(t, x)=0, x \in \partial \Omega,
$$

and with the initial conditions

$$
n(0, x)=n_{0}(x), c(0, x)=c_{0}(x), u(0, x)=u_{0}(x), x \in \Omega
$$

*Received: December 17, 2012; accepted (in revised form): April 18, 2013. Communicated by Peter Markowich.

${ }^{\dagger}$ CMUC, Department of Mathematics, University of Coimbra, 3001-454 Coimbra, Portugal (mitvorot@mat.uc.pt).

Research partially supported by CMUC and FCT (Portugal), through European program COMPETE/FEDER. 
The model (1.1)-(1.4) with $f \equiv 0$ and $m=1$ was suggested in [17] in order to describe the combination of chemotaxis, metabolism, cell-cell signaling, buoyancy, diffusion, and mixing in water drops full of aerobic bacteria (Bacillus subtilis). The coupled system consisting of the equations of cell and oxygen balance and of the ones of fluid motion and continuity is based on general considerations from the bioconvection theory (cf. [6] and [13]). The nonlinear-diffusion variant of the model (i.e. when $m>1$ ) was proposed in [4]. In this paper, we also admit the proliferation/death term $f$ in the cell balance equation (1.1). Note that the convectionless chemotactic models taking into account the role of cell kinetics were put forward in [10] (see also the review paper [7]), and studied, for instance, in [1, 11, 12, 19, 20]. The typical kinetic terms are $f(n)=K n(1-n)$ (Fisher's kinetic term) and $f(n)=K n(1-n)(n-\alpha)$ (the Allee effect).

The known mathematical treatments of the problem (1.1)-(1.6) (without the kinetic term) can be divided in two groups. The first one is concerned with the simpler Stokes-Keller-Segel problem lacking the nonlinear inertial term in (1.3). The local in time weak solutions are shown to exist in [9] in the case $m=1$. For $\Omega=\mathbb{R}^{2}$ and $m=1$, there is a global weak solution [5] provided $c_{0}$ or $\phi$ are small in some norms, and under certain restrictions on $k$ and $\chi$. For $\Omega=\mathbb{R}^{2}$ and $m=2$, the global weak solution exists [4] without that additional hypotheses. The same is true for bounded $\Omega, d=2$, and $m>1$ [16] or $d=3$ and $1.81<\frac{7+\sqrt{217}}{12} \leq m \leq 2$ [4], as well as for $\Omega=\mathbb{R}^{3}$ and $m=4 / 3$ with some assumptions on $k$ and $\chi$ [8]. Moreover, in the case of bounded convex $\Omega(d=2,3)$ and slightly weaker restrictions on $k$ and $\chi$, the problem admits a global weak solution for $m=1$ [21]. The eventual convergence to the homogeneous state $\left(n \equiv \int_{\Omega} n_{0} /|\Omega|, c \equiv 0, u \equiv 0\right)$ is shown in [4] for bounded $\Omega$ and $m=2$; however, this result is not entirely correct due to the presence of steady-state solutions of the form $(n \equiv 0, c \equiv$ const,$u \equiv 0)$.

The second group studies the full Navier-Stokes-Keller-Segel problem with $m=1$. The existence of local weak solutions is treated in $[2,9]$. The global weak solutions exist for $\Omega=\mathbb{R}^{2}[8]$. Moreover, if the domain $\Omega \subset \mathbb{R}^{2}$ is bounded and convex, the solution is regular and unique [21] (a similar result in the whole plane is proved in [2]). Here, more [2] or less $[8,21]$ restrictive assumptions are imposed on $k$ and $\chi$. Global solutions exist for $\Omega=\mathbb{R}^{3}$ when the initial datum is a small smooth perturbation of the steady state $\left(n_{0}=\right.$ const, 0,0$)$ ([5], strong and unique) or when $k / \chi=$ const ([2], weak).

In this paper, we consider the problem (posed in [21]) of complete classification of all $m$ which allow for global solutions. It turned out to be suitable to deal with this problem in the general setting (1.1)-(1.6) admitting the kinetic term $f$. Herewith, two cases, which we call subcritical $\left(1 \leq m \leq \frac{d+1}{3}\right)$ and supercritical $\left(m>\frac{d+1}{3}\right)$, naturally arise. In both cases, we prove existence of global weak solutions (Theorems 3.1 and 4.1). However, the choice of the kinetic function in the supercritical case enjoys wider range of possibilities: in particular, it can be zero. The typical kinetic terms mentioned above satisfy all the assumptions in both sub- and supercritical cases. In the case $\Omega=\mathbb{R}^{2}$ and $m=1$, we prove existence of a unique classical solution without the hypothesis of [2] that $k / \chi$ is almost a constant (our Theorem 4.2). Finally, in Section 5 , we show that in the supercritical case there exist weak solutions satisfying certain dissipative estimates (Theorem 5.1), and construct minimal trajectory and global attractors of the problem in the framework of [22] (our Theorem 5.2). 


\section{Preliminaries and notation}

We use the brief notations $L_{p}, W_{p}^{\beta}, H^{\beta}=W_{2}^{\beta}, p \geq 1, \beta \in \mathbb{R}$, for the Lebesgue and Sobolev spaces on $\Omega$ with values in $F=\mathbb{R}$ or $\mathbb{R}^{d}$ or $\mathbb{R}^{d^{2}}$. Parentheses denote the bilinear form

$$
(u, v)=\int_{\Omega}(u(x), v(x))_{F} d x, u, v \in L_{1} .
$$

The norm in $L_{2}$ is $\|u\|=\sqrt{(u, u)}$. The symbol $\|u\|_{l}, l \in \mathbb{N}$, will stand for the Euclidean norm in $H^{l}$.

Let $\mathcal{V}$ be the set of smooth, divergence-free, compactly supported (in $\Omega$ ) functions with values in $\mathbb{R}^{d}$. The symbols $H, V, V_{\delta}(\delta>0)$ denote the closures of $\mathcal{V}$ in $L_{2}, H^{1}, H^{\delta}$, respectively.

The symbols $C(\mathcal{J} ; E), C_{w}(\mathcal{J} ; E), L_{2}(\mathcal{J} ; E)$, etc. denote the spaces of continuous, weakly continuous, quadratically integrable, etc. functions on an interval $\mathcal{J} \subset \mathbb{R}$ with values in a Banach space $E$. A pre-norm in the Fréchet space $C([0,+\infty) ; E)$ may be defined by the formula

$$
\|v\|_{C([0,+\infty) ; E)}=\sum_{i=1}^{+\infty} 2^{-i} \frac{\|v\|_{C([0, i] ; E)}}{1+\|v\|_{C([0, i] ; E)}} .
$$

The symbol $C$ will stand for a generic positive constant that can take different values in different lines, whereas $K_{i}, i=1,2, \ldots$, will be fixed positive constants.

We set $\langle x\rangle=\sqrt{1+|x|^{2}}$ in the case $\Omega=\mathbb{R}^{d}$, and $\langle x\rangle=1$ for bounded $\Omega$.

For any scalar function $\xi$, we denote $\xi_{+}=\max (\xi, 0)$ and $\xi_{-}=\max (-\xi, 0)$.

Finally, we give here the following definition of weak solution.

Definition 2.1. A triple $(n, c, u)$ is a weak solution to the problem (1.1)-(1.6) provided

$$
\begin{gathered}
n \geq 0, c \geq 0, \\
n \in L_{\infty}\left(0, T ; L_{1}\right) \cap L_{2}\left(0, T ; L_{2}\right) \cap L_{m}\left(0, T ; L_{m}\right) \cap W_{1}^{1}\left(0, T ;\left(W_{\infty}^{1}\right)^{*}\right), \\
f(n) \in L_{1}\left(0, T ; L_{1}\right), \nabla\left(n^{m}\right) \in L_{1}\left(0, T ; L_{1}\right), \\
c \in L_{\infty}\left(0, T ; L_{\infty} \cap H^{1}\right) \cap L_{2}\left(0, T ; H^{2}\right) \cap H^{1}\left(0, T ;\left(H^{1}\right)^{*}\right), \\
u \in L_{\infty}\left(0, T ; L_{2}\right) \cap L_{2}(0, T ; V) \cap W_{1}^{1}\left(0, T ; V^{*}\right),
\end{gathered}
$$

and for any test functions $\zeta \in W_{\infty}^{1}, \theta \in H^{1}, \psi \in V$ one has

$$
\begin{gathered}
\frac{d}{d t}(n, \zeta)-(u n, \nabla \zeta)+\left(\nabla\left(n^{m}\right), \nabla \zeta\right)-(\chi(c) n \nabla c, \nabla \zeta)=(f(n), \zeta), \\
\frac{d}{d t}(c, \theta)-(u c, \nabla \theta)+(\nabla c, \nabla \theta)+(k(c) n, \theta)=0, \\
\frac{d}{d t}(u, \psi)-\sum_{i, j=1}^{d}\left(u_{i} u_{j}, \frac{\partial \psi_{j}}{\partial x_{i}}\right)+(\nabla u, \nabla \psi)+(n \nabla \phi, \psi)=0
\end{gathered}
$$

a.e. on $(0, T)$, and equalities $(1.6)$ hold in the spaces $\left(W_{\infty}^{1}\right)^{*},\left(H^{1}\right)^{*}, V^{*}$, respectively. 


\section{The supercritical case}

In the supercritical case, the existence of weak solutions is provided by the following result.

Theorem 3.1. Let $m>\frac{d+1}{3}$. Let $\phi \in L_{1}\left(0, T ; L_{1, l o c}\right)$ with $\nabla \phi \in L_{2}\left(0, T ; L_{\infty}\right)$. Let $k, \chi$, and $f$ be continuously differentiable functions, $\chi^{\prime} \geq 0, k \geq 0, k(0)=0, f(0) \geq 0$ (but $f(0)=0$ for $\Omega=\mathbb{R}^{d}$ ), and

$$
f(y) \leq f(0)+C y
$$

for $y \geq 0$.

Let $n_{0} \in L_{1} \cap L_{\max (1, m / 2)}, n_{0} \ln n_{0} \in L_{1},\langle\cdot\rangle n_{0}(\cdot) \in L_{1}, c_{0} \in H^{1} \cap L_{\infty}, n_{0} \geq 0, c_{0} \geq 0$, $u_{0} \in H$. Then problem (1.1)-(1.6) possesses a weak solution.

Proof. Let us show that a solution $(n, c, u)$ to (1.1)-(1.6) satisfies the following formal a priori bound:

$$
\begin{aligned}
\|u\|_{L_{\infty}\left(0, T ; L_{2}\right)}+\|n \ln n\|_{L_{\infty}\left(0, T ; L_{1}\right)}+\|\langle\cdot\rangle n\|_{L_{\infty}\left(0, T ; L_{1}\right)}+\|\nabla c\|_{L_{\infty}\left(0, T ; L_{2}\right)} \\
+\|\nabla u\|_{L_{2}\left(0, T ; L_{2}\right)}+\|n\|_{L_{2}\left(0, T ; L_{2}\right)}+\left\|n^{m / 2}\right\|_{L_{2}\left(0, T ; H^{1}\right)}+\|c\|_{L_{\infty}\left(0, T ; L_{\infty}\right)} \\
+\|f(n)\|_{L_{1}\left(0, T ; L_{1}\right)}+\|f(n) \ln n\|_{L_{1}\left(0, T ; L_{1}\right)}+\|c\|_{L_{2}\left(0, T ; H^{2}\right)} \leq C .
\end{aligned}
$$

Note that here and below in the proof the generic constant $C$ may depend on $T$.

Letting $\zeta \equiv 1$ in $(2.1)$, we get

$$
\frac{d}{d t}\|n(t)\|_{L_{1}}=\int_{\Omega} f(n(t, x)) d x,
$$

SO

$$
\begin{gathered}
\quad \frac{d}{d t}\|n(t)\|_{L_{1}}+\left\|f_{-}(n)\right\|_{L_{1}}=\left\|f_{+}(n)\right\|_{L_{1}} \\
\leq \int_{\Omega} f(0) d x+C\|n(t)\|_{L_{1}} \leq C\left(1+\|n(t)\|_{L_{1}}\right),
\end{gathered}
$$

whence

$$
\|n\|_{L_{\infty}\left(0, T ; L_{1}\right)}+\left\|f_{-}(n)\right\|_{L_{1}\left(0, T ; L_{1}\right)} \leq C .
$$

But

$$
\left\|f_{+}(n)\right\|_{L_{1}\left(0, T ; L_{1}\right)} \leq C\left(1+\|n\|_{L_{1}\left(0, T ; L_{1}\right)}\right) \leq C\left(1+\|n\|_{L_{\infty}\left(0, T ; L_{1}\right)}\right) .
$$

Thus,

$$
\|f(n)\|_{L_{1}\left(0, T ; L_{1}\right)} \leq C .
$$

Putting $\theta=c^{p-1}, p \geq 2$, in (2.2), we obtain

$$
\frac{1}{p} \frac{d}{d t}\|c(t)\|_{L_{p}}^{p} \leq 0
$$

and thus

$$
\|c\|_{L_{\infty}\left(0, T ; L_{p}\right)} \leq\|c(0)\|_{L_{p}}
$$


Passing to the limit as $p \rightarrow \infty$, we derive

$$
\|c\|_{L_{\infty}\left(0, T ; L_{\infty}\right)} \leq C
$$

Hence,

$$
\|\chi(c)\|_{L_{\infty}\left(0, T ; L_{\infty}\right)}+\|k(c)\|_{L_{\infty}\left(0, T ; L_{\infty}\right)} \leq C .
$$

Note that the fact of non-negativity of $c$ and $n$ is standard and follows from the parabolic comparison principle.

We now take $\zeta=1+\ln n$ in (2.1), $\theta=-\Delta c$ in (2.2), and $\psi=u$ in (2.3), arriving at

$$
\begin{gathered}
\frac{d}{d t} \int_{\Omega} n \ln n d x+\frac{4}{m}\left(\nabla\left(n^{m / 2}\right), \nabla\left(n^{m / 2}\right)\right)-(\chi(c) \nabla c, \nabla n)=(f(n), 1+\ln n), \\
\frac{1}{2} \frac{d}{d t}(\nabla c, \nabla c)+(u c, \nabla \Delta c)-(\nabla c, \nabla \Delta c)-(k(c) n, \Delta c)=0, \\
\frac{1}{2} \frac{d}{d t}(u, u)+(\nabla u, \nabla u)+(n \nabla \phi, u)=0 .
\end{gathered}
$$

Integrating by parts, we rewrite (3.11) as

$$
\begin{aligned}
& \frac{d}{d t} \int_{\Omega} n \ln n d x+\frac{4}{m}\left\|\nabla\left(n^{m / 2}\right)\right\|^{2}+\left(\chi^{\prime}(c) \nabla c, n \nabla c\right)+(\chi(c) \Delta c, n) \\
= & (f(n), 1+\ln n),
\end{aligned}
$$

and observe that

$$
\begin{aligned}
& -(u c, \nabla \Delta c) \\
= & \sum_{i, j=1}^{d}\left(\frac{\partial u_{i}}{\partial x_{j}}, c \frac{\partial^{2} c}{\partial x_{i} \partial x_{j}}\right)+\left(u_{i}, \frac{\partial c}{\partial x_{j}} \frac{\partial^{2} c}{\partial x_{i} \partial x_{j}}\right) \\
= & \sum_{i, j=1}^{d}\left(\frac{\partial u_{i}}{\partial x_{j}}, c \frac{\partial^{2} c}{\partial x_{i} \partial x_{j}}\right)+\frac{1}{2}\left(u_{i}, \frac{\partial}{\partial x_{i}}\left[\frac{\partial c}{\partial x_{j}}\right]^{2}\right)=\sum_{i, j=1}^{d}\left(\frac{\partial u_{i}}{\partial x_{j}}, c \frac{\partial^{2} c}{\partial x_{i} \partial x_{j}}\right) .
\end{aligned}
$$

Now, (3.12) reads as

$$
\frac{1}{2} \frac{d}{d t}\|\nabla c\|^{2}-\sum_{i, j=1}^{d}\left(\frac{\partial u_{i}}{\partial x_{j}}, c \frac{\partial^{2} c}{\partial x_{i} \partial x_{j}}\right)+(\Delta c, \Delta c)-(k(c) n, \Delta c)=0 .
$$

When $\Omega$ is bounded, due to classical regularity results for the Neumann problem for the Poisson equation,

$$
\|c(t)\|_{2} \leq C(\|\Delta c(t)\|+\|c(t)\|)
$$

For the whole space, integrating by parts, we obtain

$$
\|c(t)\|_{2}=\sqrt{(c, c)+\sum_{i=1}^{d}\left(\frac{\partial c}{\partial x_{i}}, \frac{\partial c}{\partial x_{i}}\right)+\sum_{i, j=1}^{d}\left(\frac{\partial^{2} c}{\partial x_{i} \partial x_{j}}, \frac{\partial^{2} c}{\partial x_{i} \partial x_{j}}\right)}
$$




$$
\begin{aligned}
& \leq \sqrt{(c, c)+2 \sum_{i=1}^{d}\left(\frac{\partial c}{\partial x_{i}}, \frac{\partial c}{\partial x_{i}}\right)+\sum_{i, j=1}^{d}\left(\frac{\partial^{2} c}{\partial x_{i} \partial x_{j}}, \frac{\partial^{2} c}{\partial x_{i} \partial x_{j}}\right)} \\
& =\sqrt{(c, c)-2(c, \Delta c)+\sum_{i, j=1}^{d}\left(\frac{\partial^{2} c}{\partial x_{i} \partial x_{i}}, \frac{\partial^{2} c}{\partial x_{j} \partial x_{j}}\right)} \\
& =\|c(t)-\Delta c(t)\| \leq\|c(t)\|+\|\Delta c(t)\| .
\end{aligned}
$$

Hence, in both cases,

$$
\|c(t)\|_{2} \leq C(\|\Delta c(t)\|+1), t \in(0, T) .
$$

Applying (3.19) and the Cauchy inequality with epsilon to (3.16), we get

$$
\frac{d}{d t}\|\nabla c\|^{2}+2 K_{1}\|c\|_{2}^{2} \leq C+K_{2}\|\nabla u\|^{2}+K_{3}\|n\|^{2} .
$$

Observe that both for $n>1$ and $n \leq 1$ (since $f$ is $C^{1}$-smooth),

$$
[f(n) \ln n]_{+} \leq C n|\ln n| .
$$

Therefore, (3.14) yields

$$
\begin{aligned}
& \frac{d}{d t} \int_{\Omega} n \ln n d x+\frac{4}{m}\left\|\nabla\left(n^{m / 2}\right)\right\|^{2}+\left\|[f(n) \ln n]_{-}\right\|_{L_{1}} \\
\leq & C+C\|n\|_{L_{1}}+C\|n \ln n\|_{L_{1}}+K_{1}\|c\|_{2}^{2}+K_{4}\|n\|^{2} .
\end{aligned}
$$

Multiply (3.13) by $2 K_{2}$ and add to (3.20) and (3.22) to obtain

$$
\begin{aligned}
& \frac{d}{d t}\|\nabla c\|^{2}+\frac{d}{d t} \int_{\Omega} n \ln n d x+K_{2} \frac{d}{d t}\|u\|^{2} \\
& \quad+K_{1}\|c\|_{2}^{2}+K_{2}\|\nabla u\|^{2}+\frac{4}{m}\left\|\nabla\left(n^{m / 2}\right)\right\|^{2}+\left\|[f(n) \ln n]_{-}\right\|_{L_{1}} \\
& \leq C+C\|n \ln n\|_{L_{1}}+K_{5}\|n\|^{2}+K_{6}\|u \nabla \phi\|^{2} .
\end{aligned}
$$

If $\Omega=\mathbb{R}^{d}$, put $\zeta(x)=\langle x\rangle$ in (2.1) (this test function is unbounded, but (2.1) still holds since we are dealing with strong solutions now):

$$
\frac{d}{d t}\|n\langle\cdot\rangle\|_{L_{1}}=(u n, \nabla\langle\cdot\rangle)+\left(n^{m}, \Delta\langle\cdot\rangle\right)+(\chi(c) n \nabla c, \nabla\langle\cdot\rangle)+(f(n),\langle\cdot\rangle) .
$$

Let us estimate the terms in the right-hand side:

$$
\begin{aligned}
(u n, \nabla\langle\cdot\rangle) & \leq C\left(\|u\|^{2}+\|n\|^{2}\right), \\
\left(n^{m}, \Delta\langle\cdot\rangle\right) & \leq C\left\|n^{m / 2}\right\|^{2}, \\
(\chi(c) n \nabla c, \nabla\langle\cdot\rangle) & \leq C\left(\|n\|^{2}+\|\nabla c\|^{2}\right), \\
(f(n),\langle\cdot\rangle) & \leq C\|\langle\cdot\rangle n\|_{L_{1}},
\end{aligned}
$$

whence

$$
3 \frac{d}{d t}\|n\langle\cdot\rangle\|_{L_{1}} \leq K_{7}\left(1+\|u\|^{2}+\|n\|^{2}+\left\|n^{m / 2}\right\|^{2}+\|\nabla c\|^{2}+\|\langle\cdot\rangle n\|_{L_{1}}\right) .
$$


If $\Omega$ is bounded and $\langle x\rangle \equiv 1,(3.29)$ is a trivial consequence of (3.4).

Let us show that

$$
\|n\|^{2}+\left\|n^{m / 2}\right\|^{2} \leq \frac{2}{\left(K_{5}+K_{7}\right) m}\left\|\nabla\left(n^{m / 2}\right)\right\|^{2}+C .
$$

Indeed, let $m \leq 2$. Let $\beta=\frac{2}{m}$ for $d=2$ and $\beta=\frac{6}{3 m-1}$ for $d=3$. In both cases $\beta<2$. Then, using the Gagliardo-Nirenberg inequality, we proceed as

$$
\begin{aligned}
\|n\|^{2}+\left\|n^{m / 2}\right\|^{2} & \leq C\left(\|n\|^{2}+\left\|n^{1 / 2}\right\|^{2}\right) \\
& =C\left(\left\|n^{m / 2}\right\|_{L_{4 / m}}^{4 / m}+\|n\|_{L_{1}}^{2}\right) \\
& \leq C+C\left\|\nabla\left(n^{m / 2}\right)\right\|^{\beta}\left\|n^{m / 2}\right\|_{L_{2 / m}}^{4 / m-\beta}+C\left\|n^{m / 2}\right\|_{L_{2 / m}}^{4 / m} \\
& =C+C\left\|\nabla\left(n^{m / 2}\right)\right\|^{\beta}\|n\|_{L_{1}}^{2-m \beta / 2}+C\|n\|_{L_{1}}^{2} \\
& \leq C\left(1+\left\|\nabla\left(n^{m / 2}\right)\right\|^{\beta}\right) \\
& \leq \frac{2}{\left(K_{5}+K_{7}\right) m}\left\|\nabla\left(n^{m / 2}\right)\right\|^{2}+C .
\end{aligned}
$$

If $m>2$, employing the $L_{p}$-interpolation, Young, and Gagliardo-Nirenberg inequalities, we have

$$
\begin{aligned}
\|n\|^{2}+\left\|n^{m / 2}\right\|^{2} & \leq C\left(\left\|n^{1 / 2}\right\|^{2}+\left\|n^{m / 2}\right\|^{2}\right) \\
& \leq C\left(1+\left\|\nabla\left(n^{m / 2}\right)\right\|^{\frac{2 m}{m+2}}\left\|n^{m / 2}\right\|_{L_{1}}^{\frac{4}{m+2}}+\left\|n^{m / 2}\right\|_{L_{1}}^{2}\right) \\
& \leq \frac{1}{\left(K_{5}+K_{7}\right) m}\left\|\nabla\left(n^{m / 2}\right)\right\|^{2}+C\left\|n^{m / 2}\right\|_{L_{1}}^{2}+C \\
& =\frac{1}{\left(K_{5}+K_{7}\right) m}\left\|\nabla\left(n^{m / 2}\right)\right\|^{2}+C\|n\|_{L_{m / 2}^{m}}^{m}+C \\
& \leq \frac{1}{\left(K_{5}+K_{7}\right) m}\left\|\nabla\left(n^{m / 2}\right)\right\|^{2}+C\|n\|_{L_{1}}^{\frac{m}{m-1}}\|n\|_{L_{m}}^{\frac{m^{2}-2 m}{m-1}}+C \\
& \leq \frac{1}{\left(K_{5}+K_{7}\right) m}\left\|\nabla\left(n^{m / 2}\right)\right\|^{2}+C\left\|n^{m / 2}\right\|^{\frac{2 m-4}{m-1}}+C \\
& \leq \frac{1}{\left(K_{5}+K_{7}\right) m}\left\|\nabla\left(n^{m / 2}\right)\right\|^{2}+\frac{1}{2}\left\|n^{m / 2}\right\|^{2}+C
\end{aligned}
$$

which implies (3.30).

Since $(n \ln n)_{-} \leq C \sqrt{n}$, it is easy to check (cf. [2] in the unbounded case) that

$$
\|n \ln n\|_{L_{1}} \leq K_{8}+2\|\langle\cdot\rangle n\|_{L_{1}}+\int_{\Omega} n \ln n d x .
$$

Adding (3.29) with (3.23), and taking into account (3.7), (3.30), and (3.33), we get

$$
\begin{aligned}
\frac{d}{d t}\|\nabla c\|^{2} & +\frac{d}{d t} \int_{\Omega} n \ln n d x+3 \frac{d}{d t}\|\langle\cdot\rangle n\|_{L_{1}}+K_{2} \frac{d}{d t}\|u\|^{2} \\
& +K_{1}\|c\|_{2}^{2}+K_{2}\|\nabla u\|^{2}+\frac{2}{m}\left\|\nabla\left(n^{m / 2}\right)\right\|^{2}+\left\|[f(n) \ln n]_{-}\right\|_{L_{1}}
\end{aligned}
$$




$$
\leq C\left(1+\|\nabla \phi\|_{L_{\infty}}^{2}\right) \times\left[1+K_{8}+\|\nabla c\|^{2}+\int_{\Omega} n \ln n d x+3\|\langle\cdot\rangle n\|_{L_{1}}+K_{2}\|u\|^{2}\right] .
$$

Gronwall's inequality and (3.33) yield

$$
\begin{aligned}
& \|\nabla c\|^{2}+\|n \ln n\|_{L_{1}}+\|\langle\cdot\rangle n\|_{L_{1}}+K_{2}\|u\|^{2} \\
\leq & 1+K_{8}+\|\nabla c\|^{2}+\int_{\Omega} n \ln n d x+3\|\langle\cdot\rangle n\|_{L_{1}}+K_{2}\|u\|^{2} \leq C,
\end{aligned}
$$

and (3.34) gives

$$
\begin{aligned}
K_{1}\|c\|_{L_{2}\left(0, T ; H^{2}\right)}^{2}+ & K_{2}\|\nabla u\|_{L_{2}\left(0, T ; L_{2}\right)}^{2} \\
& +\frac{2}{m}\left\|\nabla\left(n^{m / 2}\right)\right\|_{L_{2}\left(0, T ; L_{2}\right)}^{2}+\left\|[f(n) \ln n]_{-}\right\|_{L_{1}\left(0, T ; L_{1}\right)} \leq C .
\end{aligned}
$$

To conclude the proof of (3.2), it remains to remember (3.7), (3.21), and (3.30).

Note that

$$
\left\|\nabla\left(n^{m}\right)\right\|_{L_{1}\left(0, T ; L_{1}\right)} \leq 2\left\|\nabla\left(n^{m / 2}\right)\right\|_{L_{2}\left(0, T ; L_{2}\right)}\left\|n^{m / 2}\right\|_{L_{2}\left(0, T ; L_{2}\right)} \leq C .
$$

Now we estimate the time derivatives. Using (2.1), (3.2) and (3.37), we infer the following bound:

$$
\begin{aligned}
\int_{0}^{T}\left|\frac{d}{d t}(n, \zeta)\right| d t \leq \int_{0}^{T}|(u n, \nabla \zeta)| & \left|\left(\nabla\left(n^{m}\right), \nabla \zeta\right)\right| \\
& +|(\chi(c) n \nabla c, \nabla \zeta)|+|(f(n), \zeta)| d t \leq C\|\zeta\|_{W_{\infty}^{1}} .
\end{aligned}
$$

In the same manner we derive from $(2.2),(2.3),(3.2)$, that

$$
\begin{aligned}
& \int_{0}^{T}\left|\frac{d}{d t}(c, \theta)\right|^{2} d t \leq C\|\theta\|_{1}^{2}, \\
& \int_{0}^{T}\left|\frac{d}{d t}(u, \psi)\right| d t \leq C\|\psi\|_{1} .
\end{aligned}
$$

We still require more estimates. Firstly, let $m<2$. We find

$$
\begin{aligned}
& \|\nabla n\|_{L_{m}\left(0, T ; L_{m}\right)}^{m} \\
= & \left\||\nabla n|^{m}\right\|_{L_{1}\left(0, T ; L_{1}\right)} \\
= & \left\|\left(n^{m-1}|\nabla n|\right)^{2-m}\left(n^{\frac{m-2}{2}}|\nabla n|\right)^{2 m-2}\right\|_{L_{1}\left(0, T ; L_{1}\right)} \\
\leq & \left\|\left(n^{m-1}|\nabla n|\right)^{2-m}\right\|_{L_{\frac{1}{2-m}}}\left(0, T ; L_{\frac{1}{2-m}}\right) \\
= & \left\|\left(n^{\frac{m-2}{2}}|\nabla n|\right)^{2 m-2}\right\|_{L_{\frac{1}{m-1}}}\left(0, T ; L_{\frac{1}{m-1}}\right) \\
\leq & C\left\|\nabla\left(n^{m}\right)\right\|_{L_{L_{1}\left(0, T ; L_{1}\right)}^{2-m}\left(0, L_{1}\right)}^{2-m}\left\|\left(n^{\frac{m-2}{2}} \nabla n\right)\right\|_{L_{2}\left(0, T ; L_{2}\right)}^{2 m-2} \\
& \left.\| n^{m / 2}\right) \|_{L_{2}\left(0, T ; L_{2}\right)}^{2 m-2} \leq C .
\end{aligned}
$$


In the case $m>2$, let $\zeta=n^{\frac{m-2}{2}}$ in (2.1). Then we derive

$$
\begin{aligned}
\frac{2}{m} \frac{d}{d t}\left\|n^{m / 2}\right\|_{L_{1}} & +\frac{8 m(m-2)}{(3 m-2)^{2}}\left(\nabla\left(n^{\frac{3 m-2}{4}}\right), \nabla\left(n^{\frac{3 m-2}{4}}\right)\right) \\
& -\frac{m-2}{m}\left(\chi(c) \nabla c, \nabla\left(n^{m / 2}\right)\right)+\left(f_{-}(n), n^{\frac{m-2}{2}}\right)=\left(f_{+}(n), n^{\frac{m-2}{2}}\right) .
\end{aligned}
$$

Therefore, by the Cauchy-Bunyakovsky-Schwarz and Young inequalities,

$$
\begin{aligned}
& \frac{d}{d t}\left\|n^{m / 2}\right\|_{L_{1}}+\left\|\nabla\left(n^{\frac{3 m-2}{4}}\right)\right\|^{2}+\left(f_{-}(n), n^{\frac{m-2}{2}}\right) \\
\leq & C\left[\|\nabla c\|^{2}+\left\|\nabla\left(n^{m / 2}\right)\right\|^{2}+\left\|n^{m / 2}\right\|_{L_{1}}+\int_{\Omega} f(0)^{m / 2} d x\right] .
\end{aligned}
$$

Gronwall's lemma and (3.2) imply

$$
\left\|n^{m / 2}\right\|_{L_{\infty}\left(0, T ; L_{1}\right)}+\left\|\nabla\left(n^{\frac{3 m-2}{4}}\right)\right\|_{L_{2}\left(0, T ; L_{2}\right)}+\left\|f(n) n^{\frac{m-2}{2}}\right\|_{L_{1}\left(0, T ; L_{1}\right)} \leq C .
$$

We find, via a reasoning similar to (3.32), that

$$
\left\|n^{\frac{3 m-2}{4}}\right\|_{L_{2}\left(0, T ; L_{2}\right)} \leq C\left\|\nabla\left(n^{\frac{3 m-2}{4}}\right)\right\|_{L_{2}\left(0, T ; L_{2}\right)}+C \leq C .
$$

Now, test (2.1) by the function $\zeta n^{\frac{m-2}{2}}, \zeta \in W_{\infty}^{1}$ :

$$
\begin{aligned}
& \frac{2}{m}\left(\frac{d}{d t} n^{m / 2}, \zeta\right)+\frac{4 m}{3 m-2}\left(\nabla\left(n^{\frac{3 m-2}{4}}\right), n^{\frac{3 m-2}{4}} \nabla \zeta\right) \\
& \quad+\frac{8 m(m-2)}{(3 m-2)^{2}}\left(\nabla\left(n^{\frac{3 m-2}{4}}\right), \zeta \nabla\left(n^{\frac{3 m-2}{4}}\right)\right) \\
& -\frac{m-2}{m}\left(\chi(c) \nabla c, \zeta \nabla\left(n^{m / 2}\right)\right)-\left(\chi(c) \nabla c, n^{m / 2} \nabla \zeta\right)=\left(f(n), \zeta n^{\frac{m-2}{2}}\right) .
\end{aligned}
$$

Using (3.2), (3.44), (3.45), it is easy to deduce from (3.46) that

$$
\int_{0}^{T}\left|\frac{d}{d t}\left(n^{m / 2}, \zeta\right)\right| d t \leq C\|\zeta\|_{W_{\infty}^{1}} .
$$

Note that (3.38) is equivalent to (3.47) for $m=2$.

Having bounds (3.2), (3.37)-(3.41), (3.44), and (3.47) in hand, we can prove the existence of a weak solution via approximation of (1.1)-(1.6) by a more regular problem, and consequently pass to the limit. We omit a major part of the details (see $[2,4,5,16]$ for similar issues), and restrict ourselves to the peculiarities of passage to the limit in the porous-medium-like and growth terms. For definiteness, we consider the case of bounded $\Omega$ (the unbounded case is very similar; the spaces $L_{p}$ should be replaced by $\left.L_{p, l o c}\right)$.

The growth term $f$ can be approximated by a sequence of bounded functions $f_{N}=\frac{f N}{|f|+N}, N \in \mathbb{N}$. Let $\left(n_{N}, c_{N}, u_{N}\right)$ be the corresponding sequence of solutions and $(n, c, u)$ be the limit (intended to be the weak solution).

Due to (3.2), without loss of generality (passing to a subsequence, if necessary) $n_{N}^{m / 2} \rightarrow n^{m / 2}$ weakly in $L_{2}\left(0, T ; H^{1}\right)$. Assume first that $m \geq 2$. In view of (3.47), 
we can employ the Aubin-Lions-Simon lemma [15] to get $n_{N}^{m / 2} \rightarrow n^{m / 2}$ strongly in $L_{2}\left(0, T ; L_{2}\right)$ (here and below we always mean "up to a subsequence"). On the other hand, for $m<2, n_{N} \rightarrow n$ weakly in $L_{m}\left(0, T ; W_{m}^{1}\right)$ in view of (3.41) and (3.2). Due to (3.38), by the Aubin-Lions-Simon lemma we conclude that $n_{N} \rightarrow n$ strongly in $L_{m}\left(0, T ; L_{m}\right)$, whence $n_{N}^{m / 2} \rightarrow n^{m / 2}$ strongly in $L_{2}\left(0, T ; L_{2}\right)$ again. Hence, in both cases,

$$
\nabla\left(n_{N}^{m}\right)=2 n_{N}^{m / 2} \nabla\left(n_{N}^{m / 2}\right) \rightarrow 2 n^{m / 2} \nabla\left(n^{m / 2}\right)=\nabla\left(n^{m}\right)
$$

weakly in $L_{1}\left(0, T ; L_{1}\right)$.

Finally, let us show that $f_{N}\left(n_{N}\right) \rightarrow f(n)$ in $L_{1}\left(0, T ; L_{1}\right)$. By the Vitali convergence theorem, it suffices to see that $f_{N}\left(n_{N}\right) \rightarrow f(n)$ in measure on $(0, T) \times \Omega$ and $\left|f_{N}\left(n_{N}\right)\right|$ are uniformly integrable. We have $n_{N}^{m / 2} \rightarrow n^{m / 2}$ in $L_{2}\left(0, T ; L_{2}\right)$, thus $n_{N} \rightarrow n$ a.e. in $(0, T) \times \Omega$. Therefore

$$
f_{N}\left(n_{N}\right)-f(n)=-\frac{f\left(n_{N}\right)\left|f\left(n_{N}\right)\right|}{\left|f\left(n_{N}\right)\right|+N}+f\left(n_{N}\right)-f(n) \rightarrow 0
$$

a.e. and hence in measure. Due to $(3.2),\left\|f_{N}\left(n_{N}\right) \ln n_{N}\right\|_{L_{1}\left(0, T ; L_{1}\right)} \leq C$. Thus,

$$
\begin{aligned}
\int_{\left|f_{N}\left(n_{N}\right)\right|>M}\left|f_{N}\left(n_{N}\right)\right| d x d t & \leq C \sup _{\left|f_{N}\left(n_{N}\right)\right|>M}\left|\ln n_{N}\right|^{-1} \\
& \leq C \sup _{\left|f\left(n_{N}\right)\right|>M}\left|\ln n_{N}\right|^{-1} \rightarrow 0
\end{aligned}
$$

as $M \rightarrow+\infty$.

\section{The subcritical case}

The subcritical case requires an additional assumption on the kinetic function.

ThEOREM 4.1. Let $1 \leq m \leq \frac{d+1}{3}$. Suppose that

$$
f(y)+C_{f} y^{2} \leq f(0)+C y
$$

with some positive $C_{f}$ independent of $y \geq 0$, and the remaining assumptions of Theorem 3.1 hold. Then the problem (1.1)-(1.6) possesses a weak solution.

Proof. Let us describe the differences with the proof of Theorem 3.1. We still need to secure inequality (3.2). Firstly, (3.3), apart from yielding (3.4), gives

$$
\frac{d}{d t}\|n(t)\|_{L_{1}}+C_{f}\|n\|^{2} \leq C\left(1+\|n(t)\|_{L_{1}}\right)
$$

whence

$$
\|n\|_{L_{2}\left(0, T ; L_{2}\right)} \leq C
$$

Since $m \leq 2$,

$$
\left\|n^{m / 2}\right\|_{L_{2}\left(0, T ; L_{2}\right)} \leq C\left(\left\|n^{1 / 2}\right\|_{L_{2}\left(0, T ; L_{2}\right)}+\|n\|_{L_{2}\left(0, T ; L_{2}\right)}\right) \leq C .
$$

Thus, we do not need (3.30), which only holds in the supercritical case, but instead of (3.34) we have 


$$
\begin{gathered}
\frac{d}{d t}\|\nabla c\|^{2}+\frac{d}{d t} \int_{\Omega} n \ln n d x+3 \frac{d}{d t}\|\langle\cdot\rangle n\|_{L_{1}}+K_{2} \frac{d}{d t}\|u\|^{2} \\
+K_{1}\|c\|_{2}^{2}+K_{2}\|\nabla u\|^{2}+\frac{4}{m}\left\|\nabla\left(n^{m / 2}\right)\right\|^{2}+\left\|[f(n) \ln n]_{-}\right\|_{L_{1}} \\
\leq C\left(1+\|n\|^{2}+\left\|n^{m / 2}\right\|^{2}+\|\nabla \phi\|_{L_{\infty}}^{2}\right) \\
\times\left[1+K_{8}+\|\nabla c\|^{2}+\int_{\Omega} n \ln n d x+3\|\langle\cdot\rangle n\|_{L_{1}}+K_{2}\|u\|^{2}\right] .
\end{gathered}
$$

Gronwall's lemma, (4.3), (4.4), and (3.33) imply (3.35), (3.36), and (3.2).

In the whole-plane case, the problem possesses a unique global regular solution.

TheOREM 4.2. Let $\Omega=\mathbb{R}^{2}, m=1$, and assume that $f, \chi$, and $k$ are $C^{3}$-smooth, $f^{\prime}(y)+\left|f^{\prime \prime}(y)\right| \leq C$ for $y \geq 0, \nabla \phi \in W_{\infty}^{2}$ (and independent of $t$ ), $n_{0} \in H^{2}, c_{0} \in H^{3}, u_{0} \in$ $H^{3}$, and the remaining assumptions of Theorem 4.1 hold. Then there exists a unique classical solution to (1.1)-(1.6), satisfying

$$
\begin{gathered}
n \geq 0, c \geq 0, \\
n \in L_{\infty}\left(0, T ; H^{2}\right) \cap L_{2}\left(0, T ; H^{3}\right), \\
c \in L_{\infty}\left(0, T ; H^{3}\right) \cap L_{2}\left(0, T ; H^{4}\right), \\
u \in L_{\infty}\left(0, T ; H^{3}\right) \cap L_{2}\left(0, T ; H^{4}\right) .
\end{gathered}
$$

Proof. We observe that

$$
(\nabla f(n), \nabla n)=\left(f^{\prime}(n) \nabla n, \nabla n\right) \leq C\|\nabla n\|^{2},
$$

and

$$
\begin{aligned}
(\Delta f(n), \Delta n) & =\left(f^{\prime}(n) \Delta n, \Delta n\right)+\left(f^{\prime \prime}(n) \nabla n \Delta n, \nabla n\right) \\
& \leq C(\Delta n, \Delta n)+C\|\Delta n\|\|\nabla n\|_{L_{4}}^{2} \\
& \leq C\|\Delta n\|^{2}+C\|\Delta n\|^{2}\|\nabla n\| .
\end{aligned}
$$

Having this at hand, one may check that the blow-up criterion

$$
\|\nabla c\|_{L_{2}\left(0, T ; L_{\infty}\right)}=+\infty
$$

proven in [2] for $f \equiv 0$ remains valid in our situation, and in the absence of blow-up, i.e. when

$$
\|\nabla c\|_{L_{2}\left(0, T ; L_{\infty}\right)}<+\infty
$$

the solution is unique and its regularity is determined by (4.6)-(4.8). The argument showing that (4.12) follows from (3.2) is a slight variation of the one ending the proof of Theorem 1.3 in [2]. 


\section{Attractors}

In this section we study the long-time behavior of problem (1.1)-(1.5). We restrict ourselves to the supercritical case (cf. Remark 5.2 below). Since we cannot establish uniqueness of the weak solutions, we treat the question via the theory of trajectory attractors. More precisely, owing mainly to technical convenience, we use our version of the theory [22, Chapter 4] instead of the more classical approaches of ChepyzhovVishik [3] and Sell [14]. However, we do not know if the latter ones are applicable to (1.1)-(1.5).

In order to simplify the presentation, we consider the autonomous case

$$
\nabla \phi \in L_{\infty}
$$

(independent of $t$ ). However, similar results can be obtained in the non-autonomous case via employment of the more involved theory of pullback trajectory attractors developed recently in [18].

We start with recalling some basic framework from [22, Chapter 4].

Let $E$ and $E_{0}$ be Banach spaces, $E \subset E_{0}, E$ is reflexive. Fix some set

$$
\mathcal{H}^{+} \subset C\left([0,+\infty) ; E_{0}\right) \cap L_{\infty}(0,+\infty ; E)
$$

of solutions (strong, weak, etc.) for any given autonomous differential equation or boundary value problem. Hereafter, the set $\mathcal{H}^{+}$will be called the trajectory space and its elements will be called trajectories. Generally speaking, the nature of $\mathcal{H}^{+}$may be different from the just described one.

Definition 5.1. A set $P \subset C\left([0,+\infty) ; E_{0}\right) \cap L_{\infty}(0,+\infty ; E)$ is called attracting (for the trajectory space $\left.\mathcal{H}^{+}\right)$if for any set $B \subset \mathcal{H}^{+}$which is bounded in $L_{\infty}(0,+\infty ; E)$, one has

$$
\sup _{u \in B} \inf _{v \in P}\|T(h) u-v\|_{C\left([0,+\infty) ; E_{0}\right)} \underset{h \rightarrow \infty}{\rightarrow} 0 .
$$

Here $T(h)$ stands for the translation (shift) operator,

$$
T(h)(u)(t)=u(t+h) .
$$

Definition 5.2. A set $P \subset C\left([0,+\infty) ; E_{0}\right) \cap L_{\infty}(0,+\infty ; E)$ is called absorbing (for the trajectory space $\left.\mathcal{H}^{+}\right)$if for any set $B \subset \mathcal{H}^{+}$which is bounded in $L_{\infty}(0,+\infty ; E)$, there is $h \geq 0$ such that $T(t) B \subset P$ for all $t \geq h$.

Definition 5.3. A set $\mathcal{U} \subset C\left([0,+\infty) ; E_{0}\right) \cap L_{\infty}(0,+\infty ; E)$ is called the minimal trajectory attractor (for the trajectory space $\mathcal{H}^{+}$) if

i) $\mathcal{U}$ is compact in $C\left([0,+\infty) ; E_{0}\right)$ and bounded in $L_{\infty}(0,+\infty ; E)$;

ii) $T(t) \mathcal{U}=\mathcal{U}$ for any $t \geq 0$;

iii) $\mathcal{U}$ is attracting;

iv) $\mathcal{U}$ is contained in any other set satisfying conditions i), ii), iii).

Definition 5.4. A set $\mathcal{A} \subset E$ is called the global attractor (in $E_{0}$ ) for the trajectory space $\mathcal{H}^{+}$if

i) $\mathcal{A}$ is compact in $E_{0}$ and bounded in $E$; filled:

ii) for any bounded in $L_{\infty}(0,+\infty ; E)$ set $B \subset \mathcal{H}^{+}$the attraction property is ful-

$$
\sup _{u \in B} \inf _{v \in \mathcal{A}}\|u(t)-v\|_{E_{0}} \underset{t \rightarrow \infty}{\rightarrow} 0
$$


iii) $\mathcal{A}$ is the minimal set satisfying conditions i) and ii) (that is, $\mathcal{A}$ is contained in every set satisfying conditions i) and ii)).

Proposition 5.1. Assume that there exists an absorbing set $P$, which is relatively compact in $C\left([0,+\infty) ; E_{0}\right)$ and bounded in $L_{\infty}(0,+\infty ; E)$, for the trajectory space $\mathcal{H}^{+}$. Then there exists a minimal trajectory attractor $\mathcal{U}$ for the trajectory space $\mathcal{H}^{+}$.

Proposition 5.2. If there exists a minimal trajectory attractor $\mathcal{U}$ for the trajectory space $\mathcal{H}^{+}$, then there is a global attractor $\mathcal{A}$ for the trajectory space $\mathcal{H}^{+}$, and for all $t \geq 0$ one has $\mathcal{A}=\{\xi(t) \mid \xi \in \mathcal{U}\}$.

Remark 5.1. As a matter of fact, the existence of an absorbing set $P$ implies that $\mathcal{U}$ and $\mathcal{A}$ also attract the trajectories in the weak-* topology of $L_{\infty}(0,+\infty ; E)$ and weak topology of $E$, respectively. The reason is that, for any set $B \subset \mathcal{H}^{+}$which is bounded in $L_{\infty}(0,+\infty ; E)$ and large $h$, the sets $\{T(h) u \mid u \in B\}$ and $\{u(h) \mid u \in B\}$ are bounded and thus relatively weakly-* and weakly compact in $L_{\infty}(0,+\infty ; E)$ and $E$, respectively. This simple remark is important since in the applications the space $E_{0}$ can be comparatively weird.

We return to the bioconvection model, and make the following assumptions:

a) $\Omega$ is bounded.

b) $m>\frac{d+1}{3}$.

c) $\phi \in L_{1}, \nabla \phi \in L_{\infty}$.

d) $k, \chi$, and $f$ are continuously differentiable functions, $\chi^{\prime} \geq 0, k \geq 0, k(0)=0$.

e) The initial concentration of oxygen does not exceed some constant $c_{\mathcal{O}}$. This unusual assumption is necessary for the presence of a compact attractor, at least when $f(0)=0$. Indeed, without an assumption of this kind no compact attractor may exist due to the presence of steady-state solutions $\left(n \equiv 0, c \equiv c_{0}, u \equiv 0\right)$ with arbitrarily large constants $c_{0}$ independent of $x$. An alternative (which we do not like) is to fix the initial oxygen concentration, and to only let $n_{0}$ and $u_{0}$ vary.

f) There exists a positive number $\gamma$ so that

$$
f(y)+2 \gamma y \leq C, y \geq 0,
$$

Without loss of generality, we may assume that $\gamma$ is sufficiently small so that

$$
2 \gamma \leq K_{1}
$$

and

$$
4 \gamma\|u\|^{2} \leq\|\nabla u\|^{2}, u \in V .
$$

Let us specify the class of solutions to (1.1)-(1.5) to be considered within this section.

Definition 5.5. A triple $(n, c, u) \in L_{\infty}\left(0,+\infty ; L_{1} \times H^{1} \times H\right)$ is an admissible weak solution to problem (1.1)-(1.5) if it is a weak solution on each bounded interval $[0, T]$, and it satisfies the inequalities

$$
\begin{aligned}
& \|n\|_{L_{\infty}\left(t, t+1 ; L_{1}\right)}+\|n \ln n\|_{L_{\infty}\left(t, t+1 ; L_{1}\right)} \\
& +\|n\|_{L_{\infty}\left(t, t+1 ; L_{\max (1, m / 2)}\right)}^{\max (1,2)}+\|c\|_{L_{\infty}\left(t, t+1 ; H^{1}\right)}^{2}+\|u\|_{L_{\infty}(t, t+1 ; H)}^{2}
\end{aligned}
$$




$$
\begin{gathered}
+\|n\|_{L_{2}\left(t, t+1 ; L_{2}\right)}^{2}+\left\|n^{[\max (4,3 m-2)] / 2}\right\|_{L_{1}\left(t, t+1 ; L_{1}\right)}+\|c\|_{L_{2}\left(t, t+1 ; H^{2}\right)}^{2}+\|u\|_{L_{2}(t, t+1 ; V)}^{2} \\
\leq \Gamma\left[1+e^{-\gamma t}\left(\|n(0)\|_{L_{1}}+\|n(0) \ln n(0)\|_{L_{1}}+\|n(0)\|_{L_{\max (1, m / 2)}^{\max (1, m / 2)}}^{2}+\|c(0)\|_{1}^{2}+\|u(0)\|^{2}\right)\right], \\
\|c(t)\|_{L_{\infty} \leq c_{\mathcal{O}}}
\end{gathered}
$$

for all $t \geq \frac{\ln \left(\left\|n_{0}\right\|_{L_{1}}\right)}{\gamma}$, where $\Gamma$ is a certain constant depending on $\nabla \phi, k, \chi, f, c_{\mathcal{O}}, \gamma$, and $m$ (it will be defined during the proof of Theorem 5.1).

As the following proposition shows, the class of admissible weak solutions is sufficiently wide.

TheOREm 5.1. Let $\left(n_{0}, c_{0}, u_{0}\right)$ be as in Theorem 3.1, and $c_{0} \leq c_{\mathcal{O}}$. Then there exists an admissible weak solution to (1.1)-(1.5) satisfying the initial condition (1.6).

Proof. It suffices to formally establish (5.4) and (5.5) for the solutions of (1.1)(1.5), and to pass to the limit as in the proof of Theorem 3.1.

Inequality (5.5) is straightforward, giving also (3.10).

We have

$$
(f(y)+\gamma y) \ln y \leq C, y>0,
$$

and

$$
(f(y)+\gamma y) y^{p} \leq C, y \geq 0
$$

for any fixed $p>0$. These inequalities follow from the observations that, for large $y$, $f(y)+\gamma y$ is negative by virtue of (5.1), whereas, for small $y$,

$$
(f(y)+\gamma y)_{-} \leq(f(y)-f(0))_{-} \leq C y
$$

by the mean value theorem.

We deduce from (3.3) that

$$
\frac{d}{d t}\|n(t)\|_{L_{1}}+\gamma\|n(t)\|_{L_{1}} \leq C
$$

so

$$
\|n(t)\|_{L_{1}} \leq C+e^{-\gamma t}\left\|n_{0}\right\|_{L_{1}}
$$

For $t \geq \frac{\ln \left(\left\|n_{0}\right\|_{L_{1}}\right)}{\gamma}$, we have

$$
\|n(t)\|_{L_{1}} \leq C
$$

Formulas (3.14) and (5.6) imply

$$
\begin{aligned}
& \frac{d}{d t} \int_{\Omega} n \ln n d x+\frac{4}{m}\left\|\nabla\left(n^{m / 2}\right)\right\|^{2}+\gamma \int_{\Omega} n \ln n \\
\leq & C+K_{1}\|c\|_{2}^{2}+K_{4}\|n\|^{2},
\end{aligned}
$$


whereas (3.13) gives

$$
\frac{1}{2} \frac{d}{d t}\|u\|^{2}+\|\nabla u\|^{2} \leq K_{9}\|n\|^{2}+\frac{\gamma}{2}\|u\|^{2} .
$$

Multiply (5.12) by $2 K_{2} e^{\gamma t}$ and add with (3.20) and (5.11) multiplied by $e^{\gamma t}$ to get

$$
\begin{gathered}
\frac{d}{d t}\left[e^{\gamma t}\|\nabla c(t)\|^{2}\right]+\frac{d}{d t} \int_{\Omega} e^{\gamma t} n(t, x) \ln n(t, x) d x+K_{2} \frac{d}{d t}\left[e^{\gamma t}\|u(t)\|^{2}\right] \\
-\gamma e^{\gamma t}\|\nabla c(t)\|^{2}-K_{2} \gamma e^{\gamma t}\|u(t)\|^{2} \\
+K_{1} e^{\gamma t}\|c(t)\|_{2}^{2}+K_{2} e^{\gamma t}\|\nabla u(t)\|^{2}+\frac{4 e^{\gamma t}}{m}\left\|\nabla\left(n^{m / 2}\right)(t)\right\|^{2} \\
\leq C e^{\gamma t}+K_{10} e^{\gamma t}\|n(t)\|^{2}+K_{2} \gamma e^{\gamma t}\|u(t)\|^{2} .
\end{gathered}
$$

Similarly to (3.30), we see that

$$
\|n(t)\|^{2} \leq \frac{2}{K_{10} m}\left\|\nabla\left(n^{m / 2}(t)\right)\right\|^{2}+C .
$$

Taking into account (5.3) and (5.2), we conclude that

$$
\begin{aligned}
\frac{d}{d t}\left[e^{\gamma t}\|\nabla c(t)\|^{2}\right]+\frac{d}{d t} \int_{\Omega} e^{\gamma t} n(t, x) \ln n(t, x) d x+K_{2} \frac{d}{d t}\left[e^{\gamma t}\|u(t)\|^{2}\right] \\
+\frac{K_{1} e^{\gamma t}}{2}\|c(t)\|_{2}^{2}+\frac{K_{2} e^{\gamma t}}{2}\|\nabla u(t)\|^{2}+\frac{2 e^{\gamma t}}{m}\left\|\nabla\left(n^{m / 2}\right)(t)\right\|^{2} \leq C e^{\gamma t} .
\end{aligned}
$$

Integration in time implies

$$
\begin{aligned}
& e^{\gamma h}\|\nabla c(h)\|^{2}+\int_{\Omega} e^{\gamma h} n(h, x) \ln n(h, x) d x+K_{2}\left[e^{\gamma h}\|u(h)\|^{2}\right] \\
& \quad+\int_{0}^{h} \frac{K_{1} e^{\gamma t}}{2}\|c(t)\|_{2}^{2} d t+\int_{0}^{h} \frac{K_{2} e^{\gamma t}}{2}\|\nabla u(t)\|^{2} d t+\int_{0}^{h} \frac{2 e^{\gamma t}}{m}\left\|\nabla\left(n^{m / 2}\right)(t)\right\|^{2} d t \\
& \leq C \int_{0}^{h} e^{\gamma t} d t+\left\|c_{0}\right\|_{1}^{2}+\left\|n_{0} \ln n_{0}\right\|_{L_{1}}+K_{2}\left\|u_{0}\right\|^{2} .
\end{aligned}
$$

Therefore,

$$
\begin{aligned}
& \|\nabla c(h)\|^{2}+\int_{\Omega} n(h, x) \ln n(h, x) d x+K_{2}\|u(h)\|^{2} \\
\leq & C+e^{-\gamma h}\left(\left\|c_{0}\right\|_{1}^{2}+\left\|n_{0} \ln n_{0}\right\|_{L_{1}}+K_{2}\left\|u_{0}\right\|^{2}\right) .
\end{aligned}
$$

This inequality, (5.5), (5.9), and (3.33) yield

$$
\begin{aligned}
& \|c(h)\|_{1}^{2}+\|n(h) \ln n(h)\|_{L_{1}}+\|u(h)\|^{2} \\
\leq & C\left(1+e^{-\gamma h}\left(\left\|c_{0}\right\|_{1}^{2}+\left\|n_{0}\right\|_{L_{1}}+\left\|n_{0} \ln n_{0}\right\|_{L_{1}}+\left\|u_{0}\right\|^{2}\right)\right) .
\end{aligned}
$$


Integrating (5.15) from $h$ to $h+1$, we find

$$
\begin{gathered}
e^{\gamma(h+1)}\|\nabla c(h+1)\|^{2}+\int_{\Omega} e^{\gamma(h+1)} n(h+1, x) \ln n(h+1, x) d x \\
+K_{2}\left[e^{\gamma(h+1)}\|u(h+1)\|^{2}\right]+\int_{h}^{h+1} \frac{K_{1} e^{\gamma t}}{2}\|c(t)\|_{2}^{2} d t \\
+\int_{h}^{h+1} \frac{K_{2} e^{\gamma t}}{2}\|\nabla u(t)\|^{2} d t+\int_{h}^{h+1} \frac{2 e^{\gamma t}}{m}\left\|\nabla\left(n^{m / 2}\right)(t)\right\|^{2} d t \\
\leq C \int_{h}^{h+1} e^{\gamma t} d t+\|c(h)\|_{1}^{2}+\|n(h) \ln n(h)\|_{L_{1}}+K_{2}\|u(h)\|^{2} .
\end{gathered}
$$

Due to (3.33), (5.14), and (5.18), we arrive at

$$
\begin{aligned}
& e^{\gamma}\|\nabla c(h+1)\|^{2}+e^{\gamma}\|n(h+1) \ln n(h+1)\|_{L_{1}}+K_{2} e^{\gamma}\|u(h+1)\|^{2} \\
& +\frac{K_{1}}{2} \int_{h}^{h+1}\|c(t)\|_{2}^{2} d t+\frac{K_{2}}{2} \int_{h}^{h+1}\|\nabla u(t)\|^{2} d t+K_{10} \int_{h}^{h+1}\|n\|^{2} d t \\
& \leq C\left(1+e^{-\gamma h}\left(\left\|c_{0}\right\|_{1}^{2}+\left\|n_{0}\right\|_{L_{1}}+\left\|n_{0} \ln n_{0}\right\|_{L_{1}}+\left\|u_{0}\right\|^{2}\right)\right) .
\end{aligned}
$$

Let $m>2$. Then (3.42) and (5.7) imply

$$
\begin{aligned}
& \frac{2}{m} \frac{d}{d t}\|n\|_{L_{m / 2}}^{m / 2}+\gamma\|n\|_{L_{m / 2} / 2}^{m / 2}+\frac{8 m(m-2)}{(3 m-2)^{2}}\left\|\nabla\left(n^{\frac{3 m-2}{4}}\right)\right\|^{2} \\
\leq & C\left(1+\|\nabla c\|^{2}+\left\|\nabla\left(n^{m / 2}\right)\right\|^{2}\right) .
\end{aligned}
$$

This, (5.16), and (3.33) yield (by [3, p. 35])

$$
\begin{aligned}
& \|n(h)\|_{L_{m / 2}}^{m / 2} \\
& \leq e^{-\gamma m h / 2}\left\|n_{0}\right\|_{L_{m / 2}}^{m / 2}+C \int_{0}^{h} e^{\gamma m(t-h) / 2}\left(1+\|c(t)\|_{2}^{2}+\left\|\nabla\left(n^{m / 2}\right)(t)\right\|^{2}\right) d t \\
& \quad \leq e^{-\gamma h}\left\|n_{0}\right\|_{L_{m / 2} / 2}^{m / 2}+C \int_{0}^{h} e^{\gamma(t-h)}\left(1+\|c(t)\|_{2}^{2}+\left\|\nabla\left(n^{m / 2}\right)(t)\right\|^{2}\right) d t \\
& \quad \leq C\left(1+e^{-\gamma h}\left(\left\|n_{0}\right\|_{L_{m / 2} / 2}^{m / 2}+\left\|c_{0}\right\|_{1}^{2}+\left\|n_{0}\right\|_{L_{1}}+\left\|n_{0} \ln n_{0}\right\|_{L_{1}}+\left\|u_{0}\right\|^{2}\right)\right) .
\end{aligned}
$$

Now, integration of (5.21) from $h$ to $h+1$ gives

$$
\begin{aligned}
& \int_{h}^{h+1}\left\|\nabla\left(n^{\frac{3 m-2}{4}}\right)(t)\right\|^{2} d t \\
\leq & C\left(1+e^{-\gamma h}\left(\left\|n_{0}\right\|_{L_{m / 2}}^{m / 2}+\left\|c_{0}\right\|_{1}^{2}+\left\|n_{0}\right\|_{L_{1}}+\left\|n_{0} \ln n_{0}\right\|_{L_{1}}+\left\|u_{0}\right\|^{2}\right)\right) .
\end{aligned}
$$


Similarly to (3.45), we deduce

$$
\begin{aligned}
& \int_{h}^{h+1}\left\|n^{(3 m-2) / 2}(t)\right\|_{L_{1}} d t \\
\leq & C\left(1+e^{-\gamma h}\left(\left\|n_{0}\right\|_{L_{m / 2}}^{m / 2}+\left\|c_{0}\right\|_{1}^{2}+\left\|n_{0}\right\|_{L_{1}}+\left\|n_{0} \ln n_{0}\right\|_{L_{1}}+\left\|u_{0}\right\|^{2}\right)\right) .
\end{aligned}
$$

In view of $(5.9),(5.18),(5.20),(5.22),(5.24)$, and (5.3), there exists $\Gamma$ such that (5.4) holds true.

We are going to construct the minimal trajectory attractor and the global attractor for problem (1.1)-(1.5). In the sequel, we assume that

$$
|f(y)| \leq C\left(y^{m}+1\right), y \geq 0,
$$

and $m>2$. It seems that other supercritical values of $m$ can also be treated, even without (5.25), although $m=2$ may be troublesome. For this purpose, one should observe that the major part of the considerations in [22, Chapter 4] and [18] remains valid for non-reflexive $E$.

We let

$$
E=L_{m / 2} \times H^{1} \times H
$$

and

$$
E_{0}=W_{m / 2}^{-\delta} \times H^{1-\delta} \times V_{\delta}^{*}
$$

where $\delta \in(0,1]$ is a fixed number. The trajectory space $\mathcal{H}^{+}$is the set of all admissible weak solutions to $(1.1)-(1.5)$. It is contained in $L_{\infty}(0,+\infty ; E)$. Moreover, without loss of generality we may assume that it is contained in $C\left([0,+\infty) ;\left(W_{\infty}^{1}\right)^{*} \times\left(H^{1}\right)^{*} \times V^{*}\right)$. By the Lions-Magenes lemma [22, Lemma 2.2.6], $L_{\infty}(0,+\infty ; E) \cap C\left([0,+\infty) ;\left(W_{\infty}^{1}\right)^{*} \times\right.$ $\left.\left(H^{1}\right)^{*} \times V^{*}\right) \subset C_{w}([0,+\infty) ; E)$. Since the embedding $E \subset E_{0}$ is compact, $\mathcal{H}^{+}$lies in $C\left([0,+\infty) ; E_{0}\right)$.

Lemma 5.1. The time derivatives of admissible weak solutions satisfy the estimate

$$
\begin{aligned}
& \quad\left\|n^{\prime}\right\|_{L_{\frac{3}{2}-\frac{1}{m}}\left(t, t+1 ; W_{1}^{-2}\right)}+\left\|c^{\prime}\right\|_{L_{2}\left(t, t+1 ;\left(H^{1}\right)^{*}\right)}^{2}+\left\|u^{\prime}\right\|_{L_{4 / 3}\left(t, t+1 ; V^{*}\right)}^{2} \\
& \leq \Psi\left(\|n\|_{L_{2}\left(t, t+1 ; L_{2}\right)},\|n\|_{L_{(3 m-2) / 2}\left(t, t+1 ; L_{(3 m-2) / 2}\right)},\right. \\
& \left.\|c\|_{L_{\infty}\left(t, t+1 ; H^{1}\right)},\|u\|_{L_{\infty}(t, t+1 ; H)},\|u\|_{L_{2}(t, t+1 ; V)}\right)
\end{aligned}
$$

with some continuous function $\Psi$ independent of $t \geq 0$.

We omit the proof since it is not involved (cf. [22]).

TheOREM 5.2. The trajectory space $\mathcal{H}^{+}$possesses a minimal trajectory attractor and a global attractor.

Proof. Due to propositions 5.1 and 5.2, it suffices to find an absorbing set (which is relatively compact in $C\left([0,+\infty) ; E_{0}\right)$ and bounded in $\left.L_{\infty}(0,+\infty ; E)\right)$ for the trajectory space $\mathcal{H}^{+}$. Consider the set $P$ of all triples

$$
(n, c, u) \in C\left([0,+\infty) ; E_{0}\right) \cap L_{\infty}(0,+\infty ; E)
$$


such that (5.26) and

$$
\begin{gathered}
\|n\|_{L_{\infty}\left(t, t+1 ; L_{1}\right)}+\|n \ln n\|_{L_{\infty}\left(t, t+1 ; L_{1}\right)} \\
+\|n\|_{L_{\infty}\left(t, t+1 ; L_{\max (1, m / 2))}\right.}^{\max (1, m / 2)}+\|c\|_{L_{\infty}\left(t, t+1 ; H^{1}\right)}^{2}+\|u\|_{L_{\infty}(t, t+1 ; H)}^{2} \\
+\|n\|_{L_{2}\left(t, t+1 ; L_{2}\right)}^{2}+\left\|n^{(3 m-2) / 2}\right\|_{L_{1}\left(t, t+1 ; L_{1}\right)} \\
+\|c\|_{L_{2}\left(t, t+1 ; H^{2}\right)}^{2}+\|u\|_{L_{2}(t, t+1 ; V)}^{2} \leq 2 \Gamma
\end{gathered}
$$

hold for every $t \geq 0$.

It is an absorbing set for the trajectory space $\mathcal{H}^{+}$and is bounded in $L_{\infty}(0,+\infty ; E)$. By the Aubin-Lions-Simon lemma, the set $\left\{\left.y\right|_{[0, M]}, y \in P\right\}$ is relatively compact in $C\left([0, M] ; E_{0}\right)$ for any $M>0$. This implies (cf. $[22$, p. 183$]$ ) that $P$ is relatively compact in $C\left([0,+\infty) ; E_{0}\right)$.

REMARK 5.2. Observe that (4.1) implies (5.1) for all positive $\gamma$, in particular, for the ones at which (5.3) and (5.2) hold true. Thus, one can expect existence of attractors in the subcritical case. We leave it as an open problem.

\section{REFERENCES}

[1] M. Aida, T. Tsujikawa, M. Efendiev, A. Yagi, and M. Mimura, Lower estimate of the attractor dimension for a chemotaxis growth system, J. London Math. Soc., 74(2), 453-474, 2006.

[2] M. Chae, K. Kang, and J. Lee, Existence of smooth solutions to coupled chemotaxis-fluid equations, Disc. Cont. Dyn. Sys., 33(6), 2271-2297, 2013.

[3] V.V. Chepyzhov and M.I. Vishik, Attractors for Equations of Mathematical Physics, American Mathematical Society, Providence, RI, 2002.

[4] M. Di Francesco, A. Lorz, and P. Markowich, Chemotaxis-fluid coupled model for swimming bacteria with nonlinear diffusion: Global existence and asymptotic behavior, Disc. Cont. Dyn. Sys., 28(4), 1437-1453, 2010.

[5] R. Duan, A. Lorz, and P. Markowich, Global solutions to the coupled chemotaxis-fluid equations, Commun. Part. Diff. Equ., 35(9), 1635-1673, 2010.

[6] N. Hill and T. Pedley, Bioconvection, Fluid Dyn. Res., 37(1-2), 1-20, 2005.

[7] T. Hillen and K.J. Painter, A user's guide to PDE models for chemotaxis, J. Math. Biol., 58(1-2), 183-217, 2009.

[8] J.G. Liu and A. Lorz, A coupled chemotaxis-fluid model: Global existence, Ann. Inst. H. Poincaré Anal. Non Linéaire, 28, 643-652, 2011.

[9] A. Lorz, Coupled chemotaxis fluid model, Math. Mode. Meth. Appl. Sci., 20(6), 987-1004, 2010.

[10] M. Mimura and T. Tsujikawa, Aggregating pattern dynamics in a chemotaxis model including growth, Physica A: Stat. Theo. Phys., 230(3-4), 499-543, 1996.

[11] G. Nadin, B. Perthame, and L. Ryzhik, Traveling waves for the Keller-Segel system with Fisher birth terms, Interfaces Free Bound., 10(4), 517-538, 2008.

[12] K. Osaki, T. Tsujikawa, A. Yagi, and M. Mimura, Exponential attractor for a chemotaxisgrowth system of equations, Nonlinear Anal., Ser. A: Theory Methods, 51(1), 119-144, 2002.

[13] T. Pedley and J. Kessler, Hydrodynamics phenomena in suspensions of swimming microorganisms, Annu. Rev. Fluid Mech., 24, 313-358, 1992.

[14] G.R. Sell, Global attractors for the three-dimensional Navier-Stokes equations, J. Dynam. Diff. Equ., 8(1), 1-33, 1996.

[15] J. Simon, Compact sets in the space $L^{p}(0, T ; B)$, Ann. Mat. Pura Appl., (4), 146, 65-96, 1987.

[16] Y. Tao and M. Winkler, Global existence and boundedness in a Keller-Segel-Stokes model with arbitrary porous medium diffusion, Disc. Cont. Dyn. Sys., 32(5), 1901-1914, 2012.

[17] I. Tuval, L. Cisneros, C. Dombrowski, C. Wolgemuth, J. Kessler, and R. Goldstein, Bacterial swimming and oxygen transport near contact lines, Proc. Natl. Acad. Sci. USA, 102(7), 2277-2282, 2005.

[18] D. Vorotnikov, Asymptotic behavior of the non-autonomous 3D Navier-Stokes problem with coercive force, J. Diff. Equ., 251(8), 2209-2225, 2011.

[19] Z. Wang and T. Hillen, Classical solutions and pattern formation for a volume filling chemotaxis model, Chaos, 17(3), 037108, 13, 2007. 
[20] M. Winkler, Boundedness in the higher-dimensional parabolic-parabolic chemotaxis system with logistic source, Commun. Part. Diff. Equ., 35(8), 1516-1537, 2010.

[21] M. Winkler, Global large-data solutions in a chemotaxis- Navier-Stokes system modeling cellular swimming in fluid drops, Commun. Part. Diff. Equ., 37, 319-351, 2012.

[22] V.G. Zvyagin and D.A. Vorotnikov, Topological Approximation Methods for Evolutionary Problems of Nonlinear Hydrodynamics, Walter de Gruyter \& Co., Berlin, 2008. 